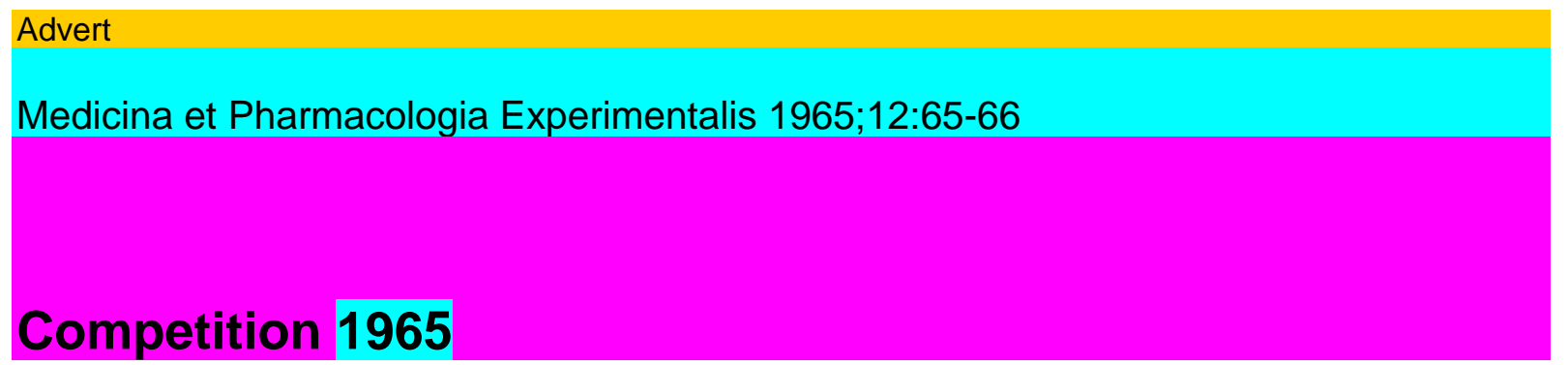

The Dr. Heinz Karger Memorial Foundation invites the submission of original papers on the following subject:

MICROANGIOLOGICAL PROBLEMS

IN ARTERIOSCLEROSIS

to compete for an award of sFr. 3000.-. The Council of the Foundation will judge the papers and confer the prize. Conditions Language: English, German or French Length of Manuscript: The manuscript shall not exceed 20 typewritten pages, including illustrations, tables and bibliography. The manuscript must be typewritten on one side only, double-spaced; it is to be submitted in duplicate and in accordance with the instructions contained in the "Rules for the Preparation of Manuscripts and Bibliographies". The booklet can be obtained from the publishers

free of charge if the request is marked "Competition". Submission date: Manuscripts marked "Competition" must reach the publishers, S. Karger AG, Arnold-Böcklin-Strasse 25, 4000 Basel 11

(Switzerland), not later than August 31, 1965.

Publication: The winning paper will be published in 1965 in one of the Karger journals.

Preisausschreiben 1965

Die Dr. Heinz Karger-Gedächtnis-Stiftung schreibt einen Preis aus für eine Originalarbeit über das Thema

MIKROANGIOLOGISCHE PROBLEME DER ARTERIOSKLEROSE

Die Höhe des Preises beträgt sFr. 3000.-. Über die Zuerkennung des

Preises entscheidet der Stiftungsrat.

Bedingungen

Sprache: Englisch, Deutsch oder Französisch.

Umfang des Manuskriptes: Es soil 20 Schreibmaschinenseiten ein-

schliesslich Abbildungen, Tabellen und Literaturverzeichnis nicht

Med. Pharmacol, exp., Vol. 12, No. 2 (1965)

:

überschreiten. Das Manuskript soil einseitig mit doppeltem Zeilen-abstand geschrieben sein, im Doppel eingereicht werden und ge-mäss unseren Richtlinien zur Abfassung von Manuskript und Lite-raturverzeichnis zusammengestellt und abgefasst sein (die Broschüre «Das Manuskript» kann beim Verlag kostenlos bezogen werden, wenn die Anfrage den Vermerk trägt «für Preisausschreiben»). Termin: Das Manuskript ist bis zum 31. August 1965 mit dem Vermerk «Preisausschreiben» an den Verlag S. Karger AG, Arnold-

Böcklin-Strasse 25, 4000 Basel 11 (Schweiz), einzusenden.

Veröjfentlichung: Die mit dem Preis ausgezeichnete Arbeit erscheint im Jahre 1965 in einer der Zeitschriften des Verlages Karger. 


\section{Concours 1965}

La Fondation Commemorative Dr. Heinz Karger récompensera d'un prix en espèces, le meilleur travail sur le theme suivant:

PROBLÈMES MICROANGIOLOGIQUES DE L'ARTÉRIOSCLÉROSE

Ce prix est fixe à fr.s. 3000.-. Le Conseil de Fondation se prononcera sur le choix du travail et de Tattribution du prix. Conditions L-angue: Anglais, allemand ou français. Volume du manuscrit: Ce dernier ne devra pas dépasser 20 pages dactylographiées, figures, tableaux et listes de references y compris. Le manuscrit est à écrire en double exemplaire et sur un côté seule-ment de chaque feuille; Гintervalle employe entre les lignes doit être double. Le manuscrit devra être rédigé conformément à nos «Directives sur la Redaction d'un Manuscrit et $\Gamma$ Etablissement d'une Bibliographie» (la brochure peut être obtenue gratuitement auprès de la Maison d'éditions, à condition que la demande porte la mention

«pour le concours »). Délai: Les travaux seront à remettre à la Maison d'éditions, S. Karger S.A., Arnold-Böcklin-Strasse 25, à 4000 Bale 11 (Suisse), jusqu' au 31 août 1965 et devront porter la remarque suivante: «pour

le concours $\gg$.

Publication: Le travail recompense sera publié en 1965, dans une des revues de la Maison d'éditions Karger. 